\title{
Reconocido, pero no guardado. Un escrito de Thomas Buchheim sobre el problema de Dios en Robert Spaemann
}

\author{
SARA GALLARDO GONZÁLEZ Y MARÍA LUISA PRO VELASCO \\ Universidad Católica de Ávila
}

\begin{abstract}
RESUMEN: El objetivo principal de este trabajo es dar a conocer en lengua castellana un texto del profesor Thomas Buchheim acerca de la «última prueba» de la existencia de Dios a partir del futurum exactum de su maestro, Robert Spaemann. Para ello se analiza en primer lugar el argumento del futurum exactum del «haber-sido». Seguidamente se exponen las objeciones contra el argumento presentado de esta forma. A continuación, la presentación del argumento a partir del futurum exactum de la verdad. Finalmente se plantean los rasgos personales de la capacidad de verdad que dan pie a considerar la necesidad de una conciencia personal para poder hablar de verdad en sentido propio.
\end{abstract}

PALABRAS CLAVE: Robert Spaemann; verdad; futurum exactum; conciencia infinita.

\section{Recognized, but not saved \\ A text of Thomas Buchheim about the problem of God by Robert Spaemenn}

\begin{abstract}
The main aim of this paper is to present, in the Spanish language, text by professor Thomas Buchheim about the «ultimate proof» of God's existence in response to futurum exactum by his professor, Robert Spaemann. In the first section the «have-been» argument of the futurm exactum is analyzed. This section is followed by the criticisms of the afore mentioned argument. Then a discussion about truth, based on the futurum exactum, is explored. Finally an analysis of the personal traits of the capacity of truth is presented, which leads to an examination of the necessity of personal awareness in order to be able to discuss truth.
\end{abstract}

KEY WORDS: Robert Spaemann; truth; futurum exactum; infinite awareness.

En «Reconocido, pero no guardado» estamos ante un texto de Thomas Buchheim ${ }^{1}$ en el que analiza una propuesta de Robert Spaemann para demostrar la existencia de Dios. Se encuentra en la obra conjunta editada por HannsGregor Nissing titulada Cualidades fundamentales de la persona. Dimensiones de lo humano según Robert Spaemann, publicada en 2008. ${ }^{2}$ Si bien es este un libro que recoge reflexiones en torno a la filosofía de Robert Spaemann (1927), no obstante, algunas de ellas, tal como es el caso del presente capítulo, son críticas abiertas a su pensamiento.

1 Thomas Bucheim (1957), es Catedrático de Metafísica y Ontología en la Facultad de Filosofía de la Ludwig-Maximilians-Universität de München.

2 H.-G. Nissing (ed.), Grundvollzüge der Person. Dimensionen des Menchseins bei Robert Spaemann (Institut zur Förderung der Glaubenslehre, München, 2008): "Erkannt, aber nicht aufbewahrt Die Person, die Erfassung des Wahren und Robert Spaemanns Gottesbeweis aus dem futurum exactum" ["Reconocido, pero no guardado. La persona, la comprensión de lo verdadero y la demostración de la existencia de Dios a partir del futurum exactum de Robert Speamann"], pp. 37-53 
En «Reconocido, pero no guardado» se ponen en cuestión algunas de las premisas sostenidas por su maestro Robert Spaemann en el libro La última demostración de la existencia de Dios ${ }^{3}$ más concretamente, en el capítulo titulado «La razonabilidad de la fe en Dios». En él Spaemann planteaba lo que pretendía que fuese una «última prueba» para la existencia de Dios, añadida a las que otros muchos pensadores han presentado a lo largo de toda la historia de la filosofía. Sin embargo, su intento parece excesivamente pretencioso tras la Modernidad y la reciente Postmodernidad o, al menos, así lo juzga su discípulo.

Ya en otras ocasiones Spaemann había planteado su argumento sobre el futurum exactum. Recordemos aquí sus exposiciones anteriores en la Escuela superior de filosofía de Múnich, en un artículo del periódico alemán Die Welt a finales de 2004, y en la lectio inauguralis de ASSET (Alta Scuola Società Economia Teologia, actualmente Studium Generale Marcianum) un centro de investigación del Patriarcado de Venecia, en 2009. Ahora bien, el planteamiento en Der letzte Gottesbeweis contiene una serie de premisas que no están contenidas en esta conferencia, y que son las que han dado lugar al artículo de Buchheim, que escribe con la intención de rebatirlas. Esta conferencia de Spaemann ha aparecido traducida en la revista Humanitas, pero el texto más completo es el que recoge la prueba que nos ocupa y cuya traducción elaborada por Marisa Pro, aparecerá en breve en la revista Cauriensia ${ }^{4}$.

En líneas generales, para el profesor Buchheim, podemos pensar y sabernos seres capaces de verdad sin necesidad de aceptar el argumento del futurum exactum o, lo que es lo mismo, del futuro anterior de la verdad. Según Spaemann, lo que ahora es verdad, o bien se eleva al grado de verdad eterna o bien el estado de cosas al que nos referimos no habrá sido en absoluto. En otras palabras: todo instante actual habrá sido verdad en el futuro. Dado que la verdad precisa de una conciencia que la recuerde, y la conciencia humana es finita, Spaemann alude a la necesidad de la existencia de una conciencia eterna, o sea, a la conciencia de Dios.

Buchheim, por su parte, señala que el argumento de Spaemann no cumple lo que promete, a no ser que un determinado concepto de verdad esté ya aceptado de antemano. Por otro lado, más que de pruebas el profesor Buchheim prefiere hablar de una serie de indicios de la existencia de Dios. Y así es cómo, una vez más, se postula la realidad del Ser Absoluto en esta sociedad post-secular, en la que nada parece ser definitivo. Veamos pues por nosotros mismos si esta aclaración crítica sobre el argumento de Spaemann resulta convincente. Al lector queda el juicio personal sobre lo aquí planteado.

A continuación, presentamos la traducción del texto íntegro. Hemos mantenido las notas del original alemán, con algunas aclaraciones nuestras entre corchetes. El resto son notas explicativas que hemos considerado oportunas cuando los idiomas se distancian tanto que hacen necesaria una mayor precisión conceptual.

3 Spaemann, R., Der letzte Gottesbeweis (Pattloch, München, 2007).

4 Cauriensia, revista anual de Ciencias Eclesiásticas, 12 (2017). 


\title{
RECONOCIDO, PERO NO GUARDADO
}

\section{LA PERSONA, LA COMPRENSIÓN DE LO VERDADERO Y LA DEMOSTRACIÓN DE LA existencia de Dios a Partir del futurum exactum de Robert Spaemann}

\begin{abstract}
«En algún apartado rincón del universo centelleante, desparramado en innumerables sistemas solares, hubo una vez un astro en el que animales inteligentes inventaron el conocimiento. Fue el minuto más altanero y falaz de la "Historia Universal", pero, a fin de cuentas, sólo un minuto. Tras breves respiraciones de la naturaleza el astro se heló y los animales inteligentes hubieron de perecer» ${ }^{5}$.
\end{abstract}

El pensamiento de Robert Spaemann sostiene que ese minuto del conocimiento del que Nietzsche habla sarcástica y despectivamente, si de verdad ha sido conocimiento, no solo abarca un minuto, sino una eternidad. Según su tesis, el conocimiento solo es posible cuando, una vez que se ha alcanzado, tiene un contenido que se retiene de forma absoluta y para siempre, que es lo que de hecho pasa. Si esto es hoy realmente así, seguirá siempre siendo verdadero el que hayamos conocido algo. Ahora bien, si sigue siendo siempre verdadero lo que hemos conocido, lo seguirá siendo incluso cuando nuestro astro se haya congelado, y nosotros, animales inteligentes, nos hayamos extinguido.

Pero, ¿qué puede querer decir —se pregunta Spaemann— que un conocimiento sea verdadero y haya sido así, en lugar de que no haya sido? ¿Cómo y dónde queda pues retenido y guardado, de forma que haya una diferencia entre el ser y el no-ser de lo conocido? O bien no debería ya ser verdadero que haya sido así y no de otra manera, como lo constata hoy nuestro conocimiento; o bien debe quedar guardado todavía, que es lo que de hecho sucede y sólo por eso puede ser contenido de nuestro conocimiento. Sin embargo, si afirmamos hoy que realmente conocemos algo, no podemos pensar que en algún momento ya no será verdad [el haberlo conocido] (la primera opción). Por lo tanto, según Spaemann debemos suponer la otra opción: que esto verdadero permanecerá todavía para una conciencia infinita y absoluta, es decir para la conciencia de Dios. Con la capacidad de conocer, Dios nos da ya hoy la prenda de ese quedar guardado lo que ha pasado, pasa y pasará, y que deja atrás todo lo fugaz. Un pensamiento bello y verdaderamente seductor, consolador y sublime para todos aquellos que se saben dotados de razón y mortales, sobre una estrella fugaz en un universo en explosión.

Según escribe Spaemann, la prueba de la existencia de Dios planteada por él a partir del futurum exactum de la verdad sería, por así decirlo, «resistente a Nietzsche» ${ }^{6}$, y "el argumento a favor de la existencia de Dios más convincente —un argumento ad hominem»— destinado a «quienes quieren comprenderse

5 Nietzsche, F., Sobre verdad y mentira en sentido extramoral y otros fragmentos de filosofía del conocimiento, (Tecnos, Madrid, 2012), 21. [Referimos aquí la edición de la traducción española de este texto].

6 Spaemann, R., «Die Vernünftigkeit des Glaubens an Gott», Der letzte cit., 31. Este capítulo ha sido traducido al español en la revista Cauriensia como indicamos en la introducción. 
a sí mismos como seres libres y capaces de la verdad» ${ }^{7}$. Un argumento $a d$ hominem es un argumento que parte de una premisa que a quien la oye le gustaría tener por verdadera, porque expresa algo que le afecta directamente y le distingue —en este caso: ser alguien libre y capaz de conocer la verdad- ¿ ¿Y quién no querría esto en realidad?8.

Aun así, un argumento ad hominem no deja de ser un argumento, esto es, debe probar o bien obligar al hombre en cuestión a aceptar por razones lógicas algo más, a saber, unas conclusiones cualesquiera -si quiere tener por verdadero lo que querría atribuirse sí mismo- . Si no quiere aceptar las conclusiones, entonces para no engañarse a sí mismo tampoco debería seguir sosteniendo esas premisas. Por eso, un argumentum ad hominem necesita una forma diáfana y determinada exactamente, a partir de la cual se puedan discutir sus presupuestos y conclusiones. A mi entender, nadie hasta la fecha ha dado forma al argumento de Spaemann sobre la existencia de Dios de tal manera que se puedan fijar sus puntos clave y con ello pueda ser examinado su poder de convicción.

En primer lugar, aclararé que el argumento no cumple lo que promete, a no ser que se presuponga de antemano el concepto de verdad. En formulaciones anteriores y diversas del argumento a partir del futurum exactum del «habersido-siempre» los estados de cosas, Spaemann ha presentado un argumento similar (sin afirmar la verdad).

Pero esto no se puede admitir por razones bastante obvias. Sólo después me referiré a la versión del argumento más prometedora en mi opinión, que se basa en la sublimación de una pretensión de verdad de nuestro conocimiento y, con ello, en el futurum exactum del «haber-sido-siempre-verdad» cualquier verdad conocida.

\section{El argumento del futurum EXactum DeL «HABER-Sido» [GEWESENSEIN]}

Este argumento reza como sigue:

(1) Nos sabemos seres reales en el presente ante otra realidad (Tesis de partida: que para nosotros mismos querríamos tener por verdadera).

(2) Algo real, que nosotros comprendemos ahora como real, seguirá siendo siempre lo que ha sucedido. (Tesis de la constitución, según la cual el presente de la realidad implica la afirmación de un futurum exactum ilimitado) ${ }^{9}$.

(3) El «haber-sido» solo puede ser algo en la medida en que, también después de haber desaparecido se convierta en el pasado de un presente futuro.

7 Spaemann, R., "Gottesbeweise nach Nietzsche», en: Das unsterbliche Gerücht (KlettCotta, Stuttgart, 2007) 37-53, aquí: 53.

8 Es decir, ¿quién no querría tener tales atributos por verdaderos?. (N. del T.).

9 «El futurum exactum pertenece a la constitución del presente». (Cf. Spaemann, R., «Gottesbeweise nach Nietzsche», en: Das unsterbliche cit., 51). 
(Tesis de la transferencia, presupone que lo pasado en tanto que sucedido [Gewesenes] permanecerá intacto en cualquier instante futuro $)^{10}$.

(4) Ser el pasado de un presente posterior significa ser recordado como sucedido [Gewesenes] en ese presente. (Tesis de la conservación: la conservación de lo ocurrido requiere la existencia de una conciencia que recuerde) ${ }^{11}$.

(5) Todo presente humano es finito y en algún momento dejará de existir. (Tesis de la finitud).

Conclusión: Para que algo presente sea real para nosotros en un sentido pleno se requiere, estrictamente hablando, la suposición de una conciencia infinita en la que todo lo pasado quede conservado a modo de recuerdo.

Esto es algo claro, en mi opinión, y pertenece de hecho a nuestro ser en tanto que personas finitas: puesto que uno quiere comprenderse y poder tomarse en serio a sí mismo como persona finita, deberá tener por verdaderas las tesis (1), (2) y (5):

Somos realmente existentes y, como tales, estamos contrapuestos a lo otro existente como realmente otro, —un sí-mismo [Selbstsein] de cualquier clase aparte de nosotros- . No todo aquello que experimentamos son meros fenómenos para nosotros, sino que algunos de ellos son cosas en sí mismas e incluso viceversa: nosotros también somos «sí-mismo» para lo otro en cuestión (Tesis de partida (1)).

En segundo lugar, hay que aceptar que, en comparación con otras realidades, nuestro presente está constituido a través del reconocimiento del futurum exactum con referencia a esto otro y a nosotros mismos ${ }^{12}$. Este rasgo ontológico fundamental de lo real en su presente se puede formular de tal forma que signifique siempre la firmeza indisponible de lo real en relación con el ser o no-ser. Una vez se ha conseguido esta firmeza, ya no está a disposición

10 «Lo presente siempre seguirá siendo real como pasado del futuro presente». (Cf. Spaemann, R., Der letzte cit., 31). "El futurum exactum es la forma de eternización. En la medida en que todo lo presente es a la vez algo que habrá sido — y ello, por siempre jamáspertenece desde siempre a la dimensión de lo atemporal. Como futuro será presente, como presente se convertirá en pasado, pero como pasado seguirá existiendo en cualquier instante futuro.» ( $C f$. Spaemann, R., Personen. Versuche über den Unterschied zwischen, etwas' und ,jemand' (Klett-Cotta, Stuttgart 1996), 130).

11 «[...] con el presente consciente - y sólo puede entenderse el presente como presente consciente - también desaparece el pasado [...]. Tenemos que pensar una conciencia en la que todo lo que sucede quede recogido, una conciencia absoluta». (Cf. SPAEmann, R., Der letzte cit, 31-32). «Si renunciamos a aceptar de manera definitiva el que todos los acontecimientos del mundo queden recogidos en una interioridad divina, entonces debemos des-realizar [entwirklichen] la realidad». (Cf. SPAEMANN, R., «Gottesbeweise nach Nietzsche», en: Das unsterbliche cit., 2).

12 En alemán: «in Bezug auf dieses Andere und uns selbst», con «Andere» el profesor Buchheim se refiere tanto a otras realidades como a Dios mismo. (N. del T.). 
de posteriores referencias a ello en relación con ello [ser o no-ser]. O bien es así o bien no ha sido así. Punto. Ninguna reflexión ni cuestionamiento posterior puede cambiar en absoluto las cosas.

Algo muy distinto sucede en el caso de las alucinaciones, los sueños y las meras apariencias, ya que es posible que, a lo mejor, en una posterior reflexión o mirada retrospectiva sobre ellos, o al interpretarlos, varíe la forma en que más tarde nos refiramos a ello. Lo real por el contrario, está decidido de una vez para siempre. (Tesis de la constitución (2)).

Tercero, hay que afirmar que también la finitud y la desaparición del presente humano - aunque haya concepciones según las cuales la persona humana sería indestructible per se a pesar de su finitud, o siempre retornaría a la existencia-. Quizá todavía cabría considerar que de este modo se podría asegurar para siempre el futurum exactum de nuestro presente. Sin embargo, pertenece justamente a uno de los puntos clave del argumento de Spaemann que se presuponga la finitud y, con ella el carácter efímero de la persona mortal, que le afecta a ella misma. Lo curioso es, precisamente, que se conjeture que las personas finitas, gracias a su supuesta capacidad de referencia consciente a la realidad, reclaman legítimamente algo que, dada su constitución, supera sus propias fuerzas. (Tesis de la finitud (5)).

\section{OBJECIONES CONTRA EL ARGUMENTO PRESENTADO DE ESTA FORMA}

Tras lo dicho quedan aún por examinar las tesis (3) y (4) que, a mi juicio, por buenas razones deben ser refutadas.

Por lo que se refiere a la tesis de la transferencia (3), habría que indicar que los predicados temporales, como por ejemplo sucedido [Gewesensein], según se entiende habitualmente, caracterizan a una cosa en su propia índole mientras exista, pero no caracterizan la modalidad de su ser con vistas a la cual realizamos nuestras afirmaciones sobre tal cosa. La temporalidad pertenece al carácter predicativo del estado de cosas, no al modo como este tiene o no tiene lugar. Hay cosas y relaciones entre ellas que son tales que poseen un carácter temporal; y también existen cosas y relaciones entre esas cosas que carecen de cualquier tipo de carácter temporal. Por ejemplo, la «enseñanza» de Sócrates en relación con Platón es un estado de cosas temporal: duró de tal a tal año, y la relación de enseñanza, en general, es siempre un estado de cosas temporal. Ahora bien, la relación de «suma de dos números» es completamente atemporal. «Cinco es la suma de tres más dos» y todas las relaciones sumatorias existen sin sentido temporal alguno. De ello cabe deducir lo siguiente: en un análisis del significado de las afirmaciones, todos los términos temporales dentro de los enunciados del tipo que sean tienen que ser referidos siempre al estado de cosas afirmado y no a su ser afirmado o afirmable.

Pero esto no significa que la existencia temporalmente determinada, como la vida de un hombre, no tuviera nada que ver con la existencia o no-existencia de objetos, que puede ser sostenida en un enunciado. Cuando se dice: «Sócrates y Hegel existen o existieron realmente - a diferencia de Diotima- y han sido 
maestros famosos de la filosofía», esto significa que ambos respectivamente han existido o no existido tal como han sido caracterizados. Y, en el caso de los hombres, a su caracterización le pertenece un determinado período de la historia. Así, o bien Diotima existió en un determinado momento histórico, o bien no existió. De igual manera, o existió como una maestra de Sócrates o no existió como tal. Todas las cosas sólo pueden existir según el modo en que han sido caracterizadas y no de otro modo. Los predicados temporales forman parte de esta caracterización, pero no la existencia afirmada.

Por lo que a la tesis de la conservación (4) se refiere, en primer lugar, cabe preguntarse: si después de los dinosaurios no existió ningún ser vivo con memoria, ¿tendría que haber existido aquella conciencia eterna para retener su haber-sucedido [Gewesensein]? No parece ser objeto de disputas el que algún hecho del pasado que haya sido, en cierto sentido sea o no el pasado perteneciente a un presente posterior. Sobre esto más bien decide sólo la especie e índole del presente posterior. Este 'tiene' un pasado del que se acuerda o no.

Además, no todo lo que constituye el pasado de un presente futuro es algo sucedido [Gewesenes]. Más bien, gran parte de ello es algo no-sucedido [Nichtgewesenes]. Por ejemplo, el que un hombre no haya vuelto a casa desde la guerra, o que el homo habilis no fuese un precursor, sino más bien un competidor de los precursores de la especie homo sapiens. Se trata de hechos importantes de nuestro pasado que, sin haber sucedido jamás, son contenidos posibles de nuestra memoria. Puesto que lo futuro es siempre la medida de lo que es su pasado y, puesto que gran parte (pero no todo lo posible) de lo nosucedido entra en su memoria y así se guarda, haber-sucedido no es ni una condición necesaria ni tampoco suficiente para pretender por esa razón que sea guardado eternamente.

Querría enumerar resumidamente una vez más aquellos argumentos que me parecen contradecir las tesis 3 y 4 :

1. Los predicados temporales pertenecen al carácter de la res, no así su modalidad ontológica (tales como el ser-posible, ser-real o ser-necesario).

2. Mucho de lo sucedido de hecho se pierde más adelante por causas físicas (por ejemplo, la forma que tiene la mancha de leche en el café antes de mezclarse uniformemente con él). Si hubiera una transferencia del habersucedido [Gewesensein], entonces por razones físicas tendríamos que aceptar la existencia de Dios.

3. Lo que ha sido o bien sigue siendo todavía hoy, o bien ya no es. Por ejemplo, el sol ha existido y todavía existe hoy, mientras que el César existió, pero ya ha dejado de existir. Si hubiese una transferencia del ser qua haber-sido, entonces, por fuerza de este haber-sido, el César existiría hoy como el sol, o bien el haber-sido es en general un ser completamente distinto del ser que precisamente tiene una cosa en sí misma. Pero esto supondría multiplicar el ser de manera innecesaria. Además del ser temporal respectivo de las cosas, contaríamos en principio con una suerte 
de creación de sombras ontológicas, a saber, su haber-sido eterno. Esto sería una especie de platonismo que ciertamente podríamos denominar tesis ontológica, pero que entonces en modo alguno suministraría un argumento en favor de la existencia de Dios. Pues un haber-sido platónico, eterno, está dado eternamente «etsi non esset Deus». Ocurriría lo mismo por cierto si partiésemos por ejemplo con Frege de la existencia atemporal de los pensamientos o las proposiciones que significan lo verdadero. En ese caso, también dichas proposiciones existirían sin necesidad del apoyo del espíritu de Dios.

4. Lo no-sucedido puede ser para un presente posterior tan importante como lo sucedido. Por ejemplo, que un meteorito por poco no se haya estrellado contra la tierra hace millones de años. ¿Existe en estos casos también una transferencia de lo no-sucedido [Nichtgewesen] qua no-haber-sucedido [Nichtgewesensein]?

5. El pasado de un presente posterior, en contra de lo que parece, no es hecho por lo pasado ni se encamina sin más hacia el futuro, sino que es hecho por lo que en cada caso le sigue y por lo que, de esta forma, en primer lugar, tiene un pasado que depende de lo sucedido en un sentido absoluto, y en segundo lugar, que se interesa por qué pasado tiene. Este último tiene el poder de elegir a partir de todo lo sucedido y lo no-sucedido, un poder del que carece el pasado. Si Dios en un determinado momento creara un hombre nuevo, entonces el mundo hasta la fecha no sería el pasado de ese presente. No obstante, todo habría sido en el mundo tal como fue, y este hombre podría investigar cómo fue todo antes de que él existiera.

Si todos los hombres del mundo fueran creados de nuevo de esta manera, el resto del mundo seguiría siendo tal como era.

De ahí que, tal como muestran estos argumentos, haber-sido es independiente tanto de que el pasado de un presente posterior sea, como de que no sea. Habersido no añade lo más mínimo a lo que una cosa es y no es (ni a lo que más tarde será y no será). Por ello tampoco existe ninguna 'transferencia' del habersido al futuro a través del propio ser en cuestión. Y tampoco existe ninguna fuerza magnética transtemporal con la que el haber-sido se presente de forma privilegiada a la memoria por encima de lo no-sucedido.

Falta por lo tanto una razón por la cual lo guardado en la eterna memoria se debería extender a lo sucedido, pero no en cambio a todo lo posible nosucedido. Si sucediera esto último, entonces habría que entender como Leibniz que todos los mundos posibles existen en el entendimiento de Dios. Ahora bien, este ya no sería un argumento a favor de la existencia de Dios, sino una tesis sobre el estatus ontológico de las posibilidades. No se debe pasar por alto la cuestión de si tal tesis tiene que compartirse o no. En mi opinión, ciertamente se puede reconocer que las posibilidades no están dadas por sí mismas, ni tampoco tienen como condición un entendimiento divino, sino más bien una determinada especie y estructura de lo real. 
Las premisas (3) y (4), por lo tanto, deben ser rechazadas en esta forma del argumento.

\section{PRESENTACión DEL ARgUMENTO A PARTIR DEL FUtURUM EXACTUM DE LA VERDAD}

Los dos problemas centrales del argumento presentado en la primera forma pueden ser resueltos con mucha mayor facilidad cuando se incluye el concepto de verdad. Pues la verdad no es un predicado temporal pero, al mismo tiempo, fundamenta ante la memoria una prioridad de lo uno sobre lo otro. En primer lugar, expondré de nuevo el argumento formulado en una serie de tesis:

(1) En el presente nos es posible conocer lo que de hecho pasa. (Tesis de partida, que nos gustaría considerar verdadera acerca de nosotros mismos = Premisa $a)$.

(2) $\mathrm{Si}$ reconocemos lo que sucede realmente, entonces es verdadera la afirmación del estado de cosas reconocido. (Explicación de (1), relacionada con un supuesto caso).

(3) La afirmación verdadera de un estado de cosas implica que lo expresado con ella siempre habrá sido verdad, incluso cuando el estado de cosas en cuestión ya no exista. (Tesis de la constitución, por la cual una verdad en un determinado momento en el presente tiene que condicionar a la verdad para siempre en el futuro $=$ Premisa $b$ ).

(4) La verdad se puede definir como «adaequatio rei et intellectus». (La definición estándar de la verdad según Tomás de Aquino y otros = Premisa c).

(5) Todo lo verdadero, también en un futuro por muy lejano que sea, implica una adecuación dada entre un estado de cosas expresado y un intelecto que formula la afirmación. (Tesis de la correspondencia como conclusión de (4)).

(6) Todo intelecto humano desaparecerá en algún momento. (Tesis de la finitud: Premisa d).

Conclusión:

En tanto que afirmamos con total seriedad la verdad de un estado de cosas en el presente, tenemos que aceptar que el ser verdad de lo afirmado no lo es solo para nosotros, sino que también consta así para un intelecto eterno.

Cabría añadir que el argumento en esta forma funciona significativamente mejor que en el primer modo del que hemos tratado con anterioridad.

Pues aquí, en primer lugar, tenemos un principio de transferencia para alzar lo que ocurre por encima del tiempo en el que ocurre. Esto ciertamente lo consigue su ser-verdadero, que, como ya advirtió Tomas de Aquino, no está ya dado con el ser mismo, sino a través de una ordenación y conveniencia con 
respecto a otro, que precisa de determinadas cualificaciones. Ser-verdadero siempre expresa un modo del ente: «secundum quod consequitur unumquodque ens in ordine ad aliud» [sic] ${ }^{13}, \mathrm{y}$, dicho de manera más exacta, un orden de correspondencia o conveniencia («convenientia») con él.

Si siempre, cuando algo es verdadero, tiene que existir una relación de correspondencia, y algo que ha sido verdadero permanece siéndolo para siempre en el futuro, entonces para todo futuro tendrá que existir una relación de correspondencia con ese ser-verdadero. Al mismo tiempo, en segundo lugar, tenemos con ello un principio de selección para lo no-sucedido [Nichtgewesene] $\mathrm{y}$ que pertenece al pasado de un presente futuro. Porque, por medio de lo verdadero, es como distinguimos todo lo posible no-sucedido bien como relevante para lo sucedido, bien como no relevante. La parte de lo no-sucedido relevante para la verdad podría sin embargo ser más pequeña que todo lo posible.

El problema de la guarda [Aufbewahrung] de lo pasado y su transferencia al futuro coinciden con la teoría de la correspondencia, porque la verdad en sí presupone tanto una presentación de los estados de cosas conforme con el entendimiento, como que su correspondencia con el estado de cosas en algún momento parece implicar su correspondencia en cualquier momento.

En las reflexiones que realizaré a continuación sobre la crítica al argumento en esta forma me gustaría dejar intactas: la Premisa a (proposición (1)): que nos es posible conocer, lo que realmente de hecho pasa, así como la Premisa d (proposición (6)): que todo entendimiento humano en algún momento ya no existirá. Ya que el motivo central del argumento de Spaemann lo constituye precisamente el que, por un lado, somos capaces de conocimiento verdadero y, por otro, que sólo somos una conciencia finita, es decir, que llegará un momento en el que esta dejará de existir. La gracia del argumento está precisamente en que con el conocimiento verdadero parece que pretendemos algo de forma legítima, cuya posesión — garantizada de forma fiable — supera las capacidades inherentes a una conciencia finita. Por lo tanto se sigue que, si no queremos prescindir de una cosa, parece que tenemos que presuponer lo otro. Según la tesis de Spaemann, Nietzsche ya vio claramente esta conexión y, por ello, rechazó la pretensión de un conocimiento de la verdad como injustificada, para desenmascarar así esa hipótesis sobre la existencia de Dios o sobre un entendimiento infinito que se introduce de contrabando, como una ilusión carente de razón alguna.

13 Von Aouin, T., «Quaestiones disputatae de veritate», Von der Wahrheit (Felix Meiner Verlag, Hamurg, 1986), I 1 c. En realidad, a la cita le falta algo, pues en el original de la obra citada dice: «uno modo secundum quod consequitur unumquodque ens in se, alio modo secundum quod consequitur unum ens in ordine ad aliud». (Ibidem, 6). Y en alemán reza como sigue: "Erstens insofern sie jedem Seienden in sich genommen folgt. Zweitens insofern sie dem einem Seienden in dessen Hinordnung auf ein anderes folgt.» (Ibidem, 7). En castellano sería algo como: un modo según el cual se sigue cualquier ser en sí, otro modo según el cual se sigue un ser en orden a otro. (N. del T.). 
Comienzo con las observaciones sobre la proposición (2) del argumento («si conocemos, que es lo que realmente pasa, entonces resulta verdadera la afirmación sobre un estado de cosas conocido»). Llama la atención que se proponga aquí un estrechamiento de la comprensión de la verdad, ciertamente necesario para el argumento y, sin embargo, al mismo tiempo carente de razón en la cosa misma: verdadero, en efecto, no es solo aquello que alguien que ha conocido un estado de cosas expresa con su capacidad de juzgar.

En ciertas condiciones, también es verdadera una afirmación que es formulada o expresada sin capacidad de juzgar y sin conocimiento del estado de cosas; y, en efecto, o bien es verdadero este enunciado o su negación y, por ello, bajo cualquier circunstancia, hay una disyunción de cualquier enunciado posible que se formule y su negación (principio de bivalencia) [principio del tercero excluso]. Esta propiedad de lo verdadero, por lo tanto, no debe ser pasada por alto porque evidentemente consiste sólo en una cuestión de formulación, pero no del conocimiento de si lo verdadero es expresado o no.

La noción de verdad tiene, pues, dos significados de los cuales el argumento spaemanniano, según parece, solo puede utilizar uno, pero no el otro. La verdad es ciertamente, por un lado, el objeto formal intencional de todas las clases de conocimiento o captación de lo que es. Por otro lado, la verdad es un predicado, es decir, una caracterización de afirmaciones, enunciados o juicios de una forma determinada. A esto lo podemos denominar verdad en sentido instrumental, la cual, para decirlo una vez más, presupone solo una determinada formulación de estados de cosas, pero no un conocimiento o un intento de conocimiento de ellos. Mi tesis crítica central será que el argumento a partir del futurum exactum de la verdad solo debe presuponer el sentido instrumental, y no el significado intencional de la verdad, significado que siempre se cumple de una forma trivial en relación con un determinado enunciado, si es que alguna vez se cumple.

Dirijo ahora la atención hacia la proposición central (3) del argumento y me pregunto por el significado exacto de la siguiente idea: «Lo enunciado en una afirmación (verdadera) habrá sido siempre verdad».

(a) En primer lugar, hay que reformular de manera completamente válida el contenido de la afirmación, haciéndola pasar [de una afirmación] del estado de cosas en cuestión, captado en el enunciado, a una oración subordinada con la conjunción que, que depende del "haber-sidoverdad». Luego, tendría que significar lo mismo lo siguiente:

Siempre habrá sido verdad que el estado de cosas x existe.

Por ejemplo:

Siempre habrá sido verdad que Robert Spaemann participa en la academia de verano 'El hombre es persona'.

(b) Además, está claro que la palabra «sido» [gewesen] aquí tampoco debe hacer referencia al ser-verdad, sino de nuevo al estado de cosas 
enunciado. Pues no puede haber sido verdadero en un pasado cercano $\mathrm{y}$, sin embargo, a lo mejor, no ser verdad posteriormente; antes bien, debe permanecer siendo verdadero siempre. Empero, el estado de cosas enunciado es, naturalmente, un estado de cosas temporal, que ha tenido lugar en un momento determinado. Nuestro ejemplo de proposición se tiene que formular pues como sigue:

Siempre será verdadero que Robert Spaemann ha participado en la academia de verano 'El hombre es persona'.

O dicho en general:

Siempre será verdadero que el estado de cosas x habrá sido o habrá existido.

(c) Tomemos ahora la definición de verdad de Tomás de Aquino, que consiste en que siempre que hay lo verdadero, debe haber una correspondencia entre una cosa (el estado de cosas en cuestión) y la formulación correspondiente en el intelecto, y en esto reside, en mi opinión, el error oculto del argumento que se pondrá de manifiesto enseguida.

«Siempre habrá conveniencia entre «el estado de cosas x, tal como ha sucedido» y la formulación de «el estado de cosas x ha sucedido» en un entendimiento».

Esta última reformulación de la afirmación de partida (3) permite, otra vez, dos explicaciones diferentes, de las cuales una es innecesariamente fuerte $\mathrm{y}$, en sentido estricto, no se encuentra en armonía con los principios arriba explicados, mientras que la otra es completamente adecuada y suficiente para mantener nuestra capacidad de verdad y de conocimiento en un sentido pleno, sin tener que aceptar por ello la conclusión.

La primera explicación (demasiado fuerte) reza como sigue:

«"El estado de cosas x ha sucedido" siempre existirá por medio de un entendimiento, cuya formulación está en conveniencia con "el estado de cosas x tal como ha sucedido"».

La otra (más débil, pero en mi opinión, adecuada en general), por el contrario, reza:

Siempre existirá conveniencia entre la formulación de «el estado de cosas $\mathrm{x}$ "ha sido" » mediante un entendimiento $\mathrm{y}$ «el estado de cosas $\mathrm{x}$, tal como "ha sido"».

En esta segunda, y a mi juicio correcta, explicación, la formulación del estado de cosas constituye una condición para implicar una posible verdad. Mientras que en la primera explicación se presupone esta misma condición y de ahí otra vez se extrae una conclusión. Como recalca la misma premisa (3), la verdad como un predicado posible de los enunciados no se puede separar nunca de la forma del enunciado con que es presentado un estado de cosas (en el caso de que haya sucedido y ya no exista). El estado de cosas ciertamente ya 
no existe, pero el estado de cosas enunciado o la afirmación del estado de cosas debe ser siempre verdadero. Por lo tanto, el haber sido enunciado o formulado es la condición para la fijación de aquello que es susceptible de verdad.

La premisa (3): «la verdadera afirmación de un estado de cosas implica que lo que con ella se enuncia siempre habrá sido verdad, incluso cuando el estado de cosas en cuestión ya no exista», establece una relación conceptual entre el concepto de verdad y los estados de cosas formulados, o sea, la conexión entre lo que se ha correspondido ya una vez y lo que seguirá correspondiéndose siempre que haya una formulación equivalente. Sin embargo, no se trata de una implicación de la existencia. En las relaciones conceptuales no hay que presuponer ningún ontological commitment. De lo contrario, la afirmación «la omnipotencia de Dios implica su omnisciencia» no podría ser verdad sin presuponer la existencia de $\operatorname{Dios}^{14}$.

El argumento del futurum exactum de la verdad parte del conocimiento posible para nosotros de lo verdadero, es decir, se fundamenta en el concepto de verdad en cuanto objeto formal intencionado, en lugar de en cuanto predicado posible de determinados estados de cosas formulados; por eso, aparece con más facilidad la insinuación de que uno necesita un entendimiento cognoscente para poder aceptar la verdad de un futuro lejano. Con todo, este no es el caso, sino que lo único que se necesita es una formulación equivalente a mi actual formulación del estado de cosas.

El que (en cualquier momento) se pueda mentar una formulación equivalente del estado de cosas, no depende en absoluto de que exista en algún momento un entendimiento que proporcione esta formulación. Ya mi formulación actual del estado de cosas es, en cuanto forma lógica, la clase entera de equivalencia de tales formulaciones en cualquier momento, a no ser que el psicologismo tuviera que mantener un lugar dentro de la lógica. Así prueba mi formulación equivalente de hoy la formulabilidad del estado de cosas para todo momento, siendo indiferente si hay alguien que los realiza o no. Y la tesis de la constitución (3), según la cual se debería presuponer el futurum exactum de la verdad como verdad en el presente, es solo la afirmación de una relación conceptual entre una formulación equivalente y su correspondencia con el estado de cosas, en caso de que el enunciado sea verdadero.

Acerca de la definición de verdad (Premisa c) que hace de fundamento en la premisa (4), hay que añadir además que el argumento deja de ser concluyente si se introduce a la gran rival de esta definición, esto es, a la teoría de la coherencia de la verdad (en vez de la teoría de la adecuación). Pues la coherencia con todo el saber ya conseguido y con todas las percepciones normales se une desde un

14 O bien: el concepto de «cuadrado redondo» implica que la línea del contorno del cuadrado se encuentre a la misma distancia de la intersección de las diagonales - esta frase no podría ser verdad sin presuponer la existencia de un cuadrado redondo-. Sin embargo, justamente por ello no puede haber ningún cuadrado redondo, ya que la formulación condicional del concepto contiene una contradicción. Esta entonces no sería verdad, si la frase no fuera verdad. 
principio solo con una conexión sistemática, que existe contingentemente, del verdadero discurso.

Pero uno puede sostener cómodamente la tesis de que la teoría de la coherencia de la verdad se tiene que apoyar tanto en el momento de la adecuación de lo afirmado como verdadero (por ejemplo, ante un acceso empírico a la cosa), como viceversa: que la teoría de la adecuación de la verdad siempre tiene que presuponer una relación, en la cual la correspondencia con el estado de cosas tan solo se puede decidir cuando hay una formulación ya dada. Por lo tanto, habría que temer que una teoría de la adecuación incontaminada - como la de Tomás de Aquino_- ${ }^{15}$, presupone de entrada la coherencia entre el estado de cosas conocido y lo que Dios ha creado así y no de otra forma, y de ahí que, por supuesto, no pueda respaldar ningún argumento de la existencia de Dios que sea independiente de esta condición.

Consiguientemente, si mis reflexiones están en lo cierto, podemos comprendernos de un modo legítimo a nosotros mismos como seres capaces de verdad, incluso sin tener que mantener el argumento del futurum exactum de la verdad como un argumento ad hominem para la existencia de Dios que sea convincente. Pues nosotros mismos seríamos capaces de verdad aun cuando nunca pudiésemos reconocer con plena certeza una verdad no analítica ( $c f$. lo indicado anteriormente acerca de la premisa (2) del argumento). Pues entonces hasta podríamos proponer una formulación de los estados de cosas que se nos den y que, o ella misma o su contraria es verdadera. Sin conocimiento estaríamos en posesión de un instrumento de la verdad que puede imponernos un cierto respeto por la técnica y la actitud de los que de él se sirven.

Por lo demás, también lo considero un elemento importante, porque un disenso sobre la verdad de la fe en Dios entre nosotros los hombres no debería llevarnos a poder acusar al que niega la existencia de Dios con pretensión de verdad. La negación de Dios con pretensión de verdad es más bien consistente y por eso solo puede ser pecado como obstinación del corazón. Si el argumento a partir del futurum exactum de la verdad fuese sólido, entonces un ateo amante de la verdad, que confiesa su convicción, sería una contradictio in adiecto. Y viceversa, aquel que admite la existencia de Dios sería alguien que explicita lo que le gustaría entender por «verdad».

\section{RASGOS PERSONALES DE LA CAPACIDAD DE VERDAD}

No quisiera terminar mis reflexiones sin dedicar antes unas palabras a lo que, desde mi punto de vista, de hecho debe presuponerse si queremos atribuirnos la capacidad de verdad de un modo legítimo. Según veo, esto es posible al menos de tres formas.

15 «[...] utrum veritas principalius inveniatur in intellectu quam in rebus». (Cf. VoN Aquin, T., Quaestiones disputatae de veritate, I 2, en: Von der Wahrheit, 12. En alemán: [Findet sich Wahrheit ursprünglicher im Verstand als in den Dingen?] (Ibidem, 13). O sea: ¿Se encuentra la verdad originariamente en el entendimiento más que en las cosas? 
En primer lugar, debemos asumir un espacio de cosas y estados de cosas que sea independiente, indisponible para el sujeto que juzga, y en cuyo ser o no-serasí podemos confiar, juzgándolo con pretensión de verdad. No somos capaces de verdad si tomamos la apariencia de los fenómenos que se nos dan como algo infranqueable en nuestro camino hacia una realidad objetiva. De este modo, permanece en la línea de lo ya dicho con anterioridad y que ha sido una idea importante para la argumentación de Spaemann: que es propio de la persona finita tener que presuponer algo con pretensión de verdad, cuya constitución supera sus propias fuerzas. Sin embargo, no sabemos qué estatura ontológica tiene este espacio de cosas y estados de cosas, inalcanzable para nosotros.

Sobre todo, lo que no sabemos es si el conjunto de todos ellos es finito y efímero o si se trata de un espacio que se funde con lo infinito e imperecedero. La cualidad personal unida a la capacidad de verdad, que está unida a esta primera condición sin condición alguna, es llamada por Aristóteles pistis, esto es, la fe en el ser-así y no-de-otro-modo de lo real, que da confianza y es independiente de nosotros.

Lo segundo que tiene que presuponerse para la capacidad de verdad es un arte de formular relacionado con las cosas, que no solo se refiere a ellas, sino que también enuncia de ellas lo que corresponde o no. La red de disyunciones que cosemos por medio del intelecto debe extenderse sobre aquella capacidad de tal forma que en principio nada quede excluido de corresponderse o no con lo formulable. A través de esa red pescamos la firmeza de lo real. Esto exige la cualidad y la capacidad personal de la negación, a diferencia de la afirmación, sin la cual tampoco podría darse la capacidad de verdad.

En tercer y último lugar, no solo se debe aspirar a una correspondencia con la cosa, tal como se me aparece a mí, sino también tal como aparece a otras personas, de tal forma que incluso entre varias personas sea posible la correspondencia y la comprensión acerca de las cosas, no como aparece a alguien de cualquier otra forma, sino más bien, como ellas son en sí mismas. La capacidad de verdad individual requiere de la capacidad de entendimiento con los demás, a quienes también les es concedido tener dicha capacidad de verdad. Nadie puede por sí solo estar en posesión de una verdad incomunicable.

Por lo tanto, hay tres rasgos característicos de la persona que deben acompañar a su capacidad de verdad y a los que hay que aferrarse, si uno no quiere abandonar el interés por la verdad y la capacidad de ella: (1) poder abandonarse a un ser independiente de mí (es decir, fe); (2) poder negar (y con ello también afirmar); (3) poder entenderse con los otros sobre esto mismo. Estos tres rasgos, bien entendidos, pueden ser vistos como indicios de la existencia de Dios, pero no como demostraciones.

Pues, en primerlugar, por lo menos yo no me puedo entender a mí mismo como un principio posible del ser independiente de mí. Todo lo demás independiente de mí, excepto Dios, en tanto que finito y transitorio por naturaleza, parece sin embargo incapaz de ser un primer principio.

En segundo lugar, tal como ya demostró Hegel, la negación puede ser entendida en una originaria autorreferencialidad, como forma del Absoluto, 
por la cual no puede no existir. Lo Absoluto o lo Existente necesario es una forma clásica para expresar aquello que nosotros llamamos Dios.

Finalmente, y en tercer lugar, por ejemplo Leibniz expuso que la comprensión recíproca entre espíritus finitos solo es posible por la mediación del espíritu infinito de Dios. Así pues, que se dé una comunicación real entre varios de nosotros sobre lo que existe en la realidad representa un claro indicio de la existencia de Dios.

En realidad, para el creyente casi todo puede convertirse en un claro indicio de la existencia de Dios, al igual que, desde siempre, un ateo interesado en el debate ha sido capaz de presentar los más claros indicios como engañosos y las demostraciones más irrefutables como dependiendo de premisas increíbles para él. De este modo, los «inteligentes» entre nosotros, sean «animales» o no, aceptan que, mientras existan hombres en este mundo, nunca se formulará una demostración definitiva de la existencia de Dios, ni tampoco quedará sin contradecir una total puesta en duda de los claros indicios de su acción, para dar así la última palabra al futurum exactum.

\section{BiBLIOGRAFÍA}

Nietzsche, F. (2012). Sobre verdad y mentira en sentido extramoral y otros fragmentos de filosofía del conocimiento. Madrid: Tecnos.

Nissing, H.-G. (2008). Grundvollzüge der Person. Dimensionen des Menchseins bei Robert Spaemann. München: Institut zur Förderung der Glaubenslehre.

Spaemann, R. (2007). Der letzte Gottesbeweis. München: Pattloch.

Spaemann, R. (2010). "Pruebas de Dios tras Nietzsche», El rumor inmortal. La cuestión sobre Dios y la ilusión de la Modernidad. Madrid: Rialp.

Spaemann, R. (2007). «Gottesbeweise nach Nietzsche», en: Das unsterbliche Gerücht. Stuttgart: Klett-Cotta.

Spaemann, R. (2000). Personas: acerca de la distinción entre «algo» y «alguien». Pamplona: Eunsa.

Spaemann, R. (1996). Personen. Versuche über den Unterschied zwischen ,etwas' und ,jemand'. Stuttgart: Klett-Cotta.

Spaemann, R. (2018). La razonabilidad de la creencia en Dios, Cauriensia.

Von Aquin, T. (1986). Quaestiones disputatae de veritate, Von der Wahrheit. Hamurg: Felix Meiner Verlag.

Universidad Católica de Ávila

Facultad de Filosofía

mlpro@usal.es
Sara GaLlardo GonZÁlez

María Luisa Pro Velasco

[Artículo aprobado para publicación en enero de 2019] 